\title{
Cancer-related fatigue in patients treated with mistletoe extracts: a systematic review and meta-analysis
}

\author{
Florian Pelzer $^{1,2}\left(\right.$ Martin Loef $^{3} \cdot$ David D. Martin $^{1} \cdot$ Stephan Baumgartner ${ }^{1,2}$
}

Received: 27 June 2021 / Accepted: 15 February 2022 / Published online: 3 March 2022

(c) The Author(s) 2022

\begin{abstract}
Purpose Cancer-related fatigue remains one of the most prevalent and distressing symptoms experienced by cancer patients. Effective treatments for cancer-related fatigue are needed. The objective of this meta-analysis is to determine the impact of mistletoe extracts as a pharmacological treatment for the management of cancer-related fatigue.

Methods We included randomized clinical trials (RCTs) and non-randomized studies of interventions (NRSIs) in cancer patients. Inclusion criteria were cancer-related fatigue severity or prevalence as an outcome and testing of mistletoe extracts compared to control groups. We searched Medline (EuropePMC), Embase, the Cochrane Central Register of Controlled Trials, Clinicaltrials.gov, and opengrey.org through October 2020. We assessed the risk of bias using the Cochrane risk of bias tools for RCTs and NRSIs and conducted a meta-analysis.

Results We performed one meta-analysis with 12 RCTs, including 1494 participants, and one meta-analysis with seven retrospective NRSIs, including 2668 participants. Heterogeneity between the studies was high in both meta-analyses. Most studies had a high risk of bias. A random-effects model showed for RCTs a standardized mean difference of -0.48 (95\% confidence interval -0.82 to $-0.14 ; p=0.006)$ and for NRSIs an odds ratio of 0.36 (95\% confidence interval 0.20 to $0.66 ; p=0.0008)$. Conclusion Treatment with mistletoe extracts shows a moderate effect on cancer-related fatigue of similar size to physical activity. These results need to be confirmed by more placebo-controlled trials. Future trials should investigate different treatment durations and their effect on cancer-related fatigue in post-treatment cancer survivors.

Trial registration.

This meta-analysis has been registered under the PROSPERO registration number CRD42020191967 on October 7, 2020.
\end{abstract}

Keywords Cancer-related fatigue $\cdot$ Mistletoe extract $\cdot$ Viscum album $\cdot$ Quality of life $\cdot$ Cancer $\cdot$ Meta-analysis

\section{Introduction}

Cancer-related fatigue (CRF) is a symptom experienced by cancer patients. The most cited definition, provided by the National Comprehensive Cancer Network in the USA, describes CRF as a distressing, persistent, subjective sense of physical, emotional, and/or cognitive tiredness or exhaustion related to cancer or cancer treatment that is not proportional to recent physical activity and that interferes with

Florian Pelzer

florian.pelzer@uni-wh.de

1 Institute of Integrative Medicine, Witten/Herdecke University, Witten, Germany

2 Society for Cancer Research, Arlesheim, Switzerland

3 CHS Institut, Berlin, Germany usual functioning [1]. There is, however, no universally accepted CRF definition [2]. The pathophysiology of CRF is unclear [3], no biomarker exists for diagnosis [3], and patients find it difficult to describe the symptom [4]. CRF is therefore measured with fatigue subscales in quality of life questionnaires or with scales assessing CRF only [4].

A recent systematic review of 129 studies with 71,568 patients reported a $49 \%$ prevalence of CRF with significant heterogeneity among studies [5]. The prevalence of CRF ranged from 11 to $99 \%$. The variation of the prevalence can be attributed to the variety of scales, with differing cut-off values, and to the different probabilities of experiencing CRF depending on the cancer type and treatment stage [5]. Patients with gastrointestinal (50\%), breast (49.7\%), and lymphoma (43.3\%) cancers reported a higher CRF prevalence than patients with gynecological $(26.2 \%)$ and prostate (26.3\%) cancers [5]. Patients in late cancer stages experience 
more CRF (60.6\%) than patients with localized cancers (46.7\%) [5].

Physical activity and psychosocial interventions are currently the first-line therapy to reduce CRF [6]. Among single pharmacological treatments, to date, only methylphenidate has evidence of improving CRF, according to a metaanalysis including seven trials with a total of 661 patients [7]. There is no consensus within the European Society for Medical Oncology, however, on whether to recommend methylphenidate against CRF [6]. Effective pharmacological treatments are needed, as physical activity has only a moderate effect size $[8,9]$ and cannot be applied in all oncologic settings, e.g., in cachectic patients [6]. Combinations of non-pharmacological and pharmacological treatments are currently also examined to increase treatment effects [10].

Mistletoe extracts from European mistletoe (Viscum album L.) are used to treat CRF [11], but the evidence has not been summarized yet. Mistletoe extracts are aqueous, total plant extracts from European mistletoe, manufactured and marketed as injectable drugs with indications in oncology [12]. In most investigational settings, mistletoe extracts are injected subcutaneously two to three times a week [13]. Injected mistletoe extracts interact both with cells of the innate and adaptive immune systems [14]. Most relevant for CRF, which can be related to reduced blood cell counts and increased inflammatory markers [3], mistletoe extracts can lead to both an increase in granulocytes [14], thereby reducing risks of neutropenia-related fatigue [15] and a reduction of inflammatory markers [14], although more research in both fields is required. In clinical routine, treatment with mistletoe extracts is often individualized by adjusting the concentration, the manufacturing method, and the mistletoe type to the patient response [16]. The treatment's effectiveness in clinical routine has been assessed in 19 (NRSIs), while standardized treatments have been evaluated in randomized clinical trials (RCTs). Clinical studies have assessed the effect of mistletoe extracts on quality of life and survival, which have been reviewed in meta-analyses recently $[17,18]$. One meta-analysis showed a non-significant effect of mistletoe extracts on the fatigue subscales of quality of life questionnaires [17]. No meta-analysis, however, has assessed the impact of mistletoe extracts on CRF in NRSIs and by including all types of CRF questionnaires. The present meta-analysis will therefore determine the impact of mistletoe extracts as a pharmacological treatment for CRF in RCTs and NRSIs.

\section{Methods}

The protocol was registered on the International Prospective Register of Systematic Reviews (PROSPERO, https://www. crd.york.ac.uk/prospero; registration no. CRD42020191967) on October 7, 2020. The Preferred Reporting Items for Systematic Reviews and Meta-analyses (PRISMA) statement was followed for reporting [19].

\section{Search strategy}

Two reviewers searched independently peer-reviewed articles and grey literature published until October 15, 2020, in the databases Medline (EuropePMC), Embase, the Cochrane Central Register of Controlled Trials, Clinicaltrials.gov, opengrey.org, and in the database of the Society for Cancer Research (Verein für Krebsforschung, VfK). The VfK database is specialized in mistletoe publications and is accessible under www.vfk.ch/informationen/literatursuche/. All search strategies, performed with no limitations on publication date or language, are published in the supplementary data (Table S1). Content experts in the field were consulted for further literature suggestions (Table S1). Literature search results were saved and transferred to Endnote (Clarivate Analytics, Philadelphia, USA) for search management.

\section{Selection criteria}

Records were included if they reported RCTs or NRSIs, as NRSIs assess individualized mistletoe treatment regimens not assessed in RCTs. All included studies evaluated the impact of mistletoe extracts on CRF severity or prevalence in cancer patients via either patient- or clinician-reported outcomes. Patient-reported outcome measures that assessed "fatigue" and "tiredness" were included in the meta-analysis, as both terms are included in the CRF definition of the National Comprehensive Cancer Network. The control groups received placebo, active control, or only the treatment common to both arms. Studies were excluded in which the verum group received mistletoe extracts together with other interventions in addition to the control group's treatment.

\section{Data extraction}

Literature selection and data extraction were both performed independently by two reviewers. Reviewers discussed discrepant results until they achieved consensus. A first set of studies was included based on screening of titles and abstracts, which then underwent full-text analysis before confirmation of the inclusion. Where multiple records were reported on the same trial, only the earliest publication with the most complete reporting was included. "Near misses" excluded after full-text analysis were specifically documented together with the reason for their exclusion. Data was extracted into an Excel spreadsheet (Microsoft Corp., Redmond, USA) designed specifically for this project. Study design, the year when the study was conducted, population 
characteristics (country, number of participants at baseline and included in the analysis, mean age, cancer type), intervention (mistletoe extract type, application form, therapy duration, treatment common to both groups, adverse effects), comparator, outcome measures, effect size, and source of funding each received a coding. If CRF was evaluated via quality of life questionnaires, only the results of their fatigue subscale were included in the meta-analysis. If reported numeric data was not sufficient to calculate effect sizes, or if other non-numeric data was missing, the reviewers contacted the authors.

\section{Risk of bias assessment}

Two reviewers assessed independently the risk of bias in RCTs with the Cochrane risk-of-bias tool for randomized trials (RoB 2) and the risk of bias in NRSIs with the Risk of Bias in Non-randomized Studies of-Interventions (ROBINSI) tool [20]. All studies were assessed according to intention-to-treat-effect analysis. Reviewers discussed discrepant results until they achieved consensus.

We did not restrict the meta-analysis to studies with a low or moderate risk of bias, as most past studies with mistletoe extracts have not been blinded and therefore automatically have a high risk of bias, due to the local reaction arising at the injection site, which has not been reproduced by a placebo yet.

\section{Statistical analysis}

All meta-analyses, subgroup analyses, and sensitivity analyses were conducted using Review Manager 5.4 (The Cochrane Collaboration, London, UK). The meta-regressions, Egger's test, and Duval and Tweedie's trim-and-fill procedure were performed with R 4.0.2 (R Foundation, Vienna, Austria) including the packages dmetar (written by Mathias Harrer, Pim Cuijpers, Toshi Furukawa, and David Ebert; https://dmetar.protectlab.org/articles/dmetar.html) and meta (written by Guido Schwarzer; https://cran.r-proje ct.org/web/packages/meta/).

\section{Main outcomes}

Data from RCTs and from retrospective NRSIs were analyzed separately due to the high methodological heterogeneity of the study designs, thereby following the recommendations of the Cochrane Handbook for Systematic Reviews of Interventions regarding the inclusion of NRSI [20].

For RCTs, the effect sizes are presented as standardized mean differences (SMDs) with 95\% confidence intervals (CIs). The SMD was calculated as the mean of CRF score changes from baseline to post-intervention between the verum and the control group divided by the pooled standard deviation using Hedges' correction for small study samples. Ordinal scales reported in just one RCT [21] were dichotomized to calculate odds ratios (ORs) and subsequently converted to SMD following the Hasselblad and Hedges method $[22,23]$. For NRSIs, in contrast, the OR was the predominant outcome parameter and was therefore selected as a summary measure; published SMDs [24] were converted to ORs accordingly $[22,23]$.

The imputation of missing values is described in the supplement. If there was a choice between multiple analyses [25] or multiple time points of the outcome [24-27], a summary effect size was calculated in accordance with the literature [20]: we used an integrative approach [28] and calculated the arithmetic mean of two or more outcome values. The pooled effect estimate therefore represents an average of different types of analyses or time points of the underlying studies. To address the high heterogeneity among studies, random-effect meta-analyses were selected for the primary analysis [20].

\section{Sensitivity analysis}

Since a number of studies displayed effect estimates based on single post-intervention outcomes [21,29-32] whereas others did not [15, 33-35], we tested our handling of data multiplicity by re-calculating the meta-analyses and replaced summary effects by single outcome values resulting either from different types of analysis or from the last measurement under treatment, respectively [24-27].

Since the random-effect estimate can shift towards the smaller studies in case of a small-study effect [20], we repeated the analysis with a fixed-effect model and compared the pooled effect sizes to the outcomes of primary analysis.

The only prospective NRSI [36] was not included in the main analyses, to avoid increasing the heterogeneity by mixing prospective and retrospective designs [20]. The prospective NRSI was added in the sensitivity analysis of both prospective RCTs and retrospective NRSIs. Finally, sensitivity analyses were conducted to examine sources of heterogeneity for the NRSIs.

\section{Subgroup analyses}

We assessed the presence of heterogeneity between the studies via the Cochrane Q test and its size by the index of heterogeneity $\left(I^{2}\right)$ [37]. As a rough guide, ranges of $I^{2}$ between 30 and $60 \%, 50$ and $90 \%$, and 75 and $100 \%$ indicate moderate, substantial, and considerable heterogeneity, respectively [20]. In order to investigate possible sources of heterogeneity, we conducted subgroup analyses for multiple moderators (country, cancer type, blinding status, mistletoe extract type, control type, additional treatment, measurement instrument, risk of bias, baseline fatigue score, study size 
and intervention duration). In addition, we used meta-regressions to examine the impact of the intervention duration and baseline fatigue level on the effect size.

\section{Publication bias}

We checked for a publication bias by examining funnel plots, Egger's test [38], and Duval and Tweedie's trim-and-fill procedure [39].

\section{Results}

We identified 802 publications by electronic searches after removing duplicates. Among the 29 full-texts analyzed, 20 studies met the inclusion criteria (Fig. 1). Reasons for the exclusion of nine "near misses" are given in the supplementary data (Table S2). We contacted nine authors and institutions/manufacturers for 12 studies, of whom five granted additional information for eight studies [25, 26, 29, 30, 33, 40-42].

\section{Study description}

Twenty studies were included in the systematic review. There were 12 RCTs [15, 21, 25-27, 29-35], of which two were double-blinded [31, 32]. Out of eight NRSIs [24, 36, 40-45], seven had a retrospective and one a prospective design [36]. RCTs and NRSIs were analyzed in two separate meta-analyses.

\section{Setting and patient characteristics}

Nine studies were conducted in Germany, two in Germany and Switzerland, three in Serbia, two in Bulgaria/Russia/ Ukraine, and one in China, Italy, Israel, and South Korea respectively (Table 1). CRF was measured in eleven breast, two lung, and two pancreatic cancer studies. Gastric, head and neck, and colorectal cancer as well as osteosarcoma and
Fig. 1 PRISMA flow diagram. The reasons for excluding records after full-text assessment $\left(^{*}\right)$ are shown in Table S2

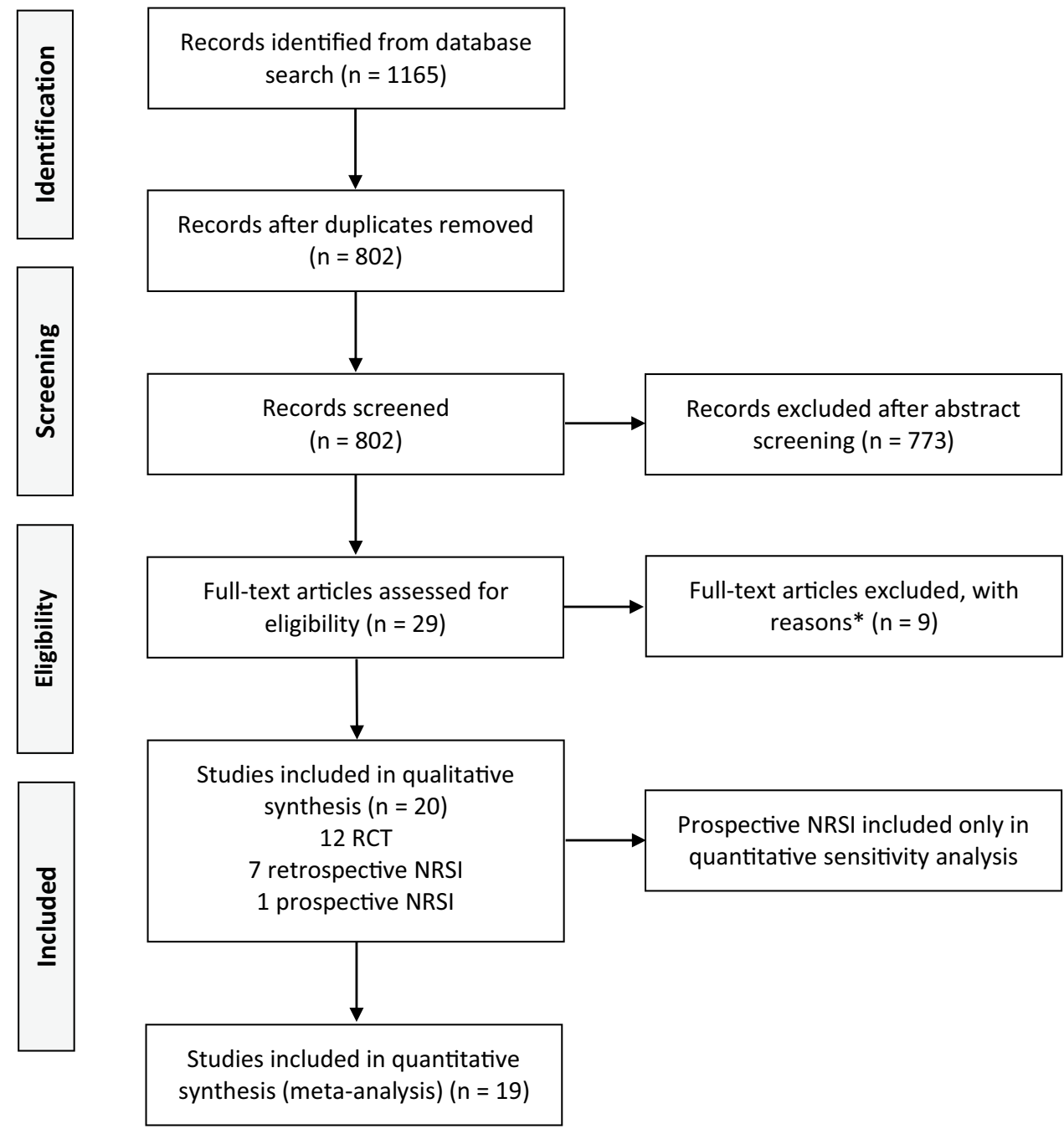




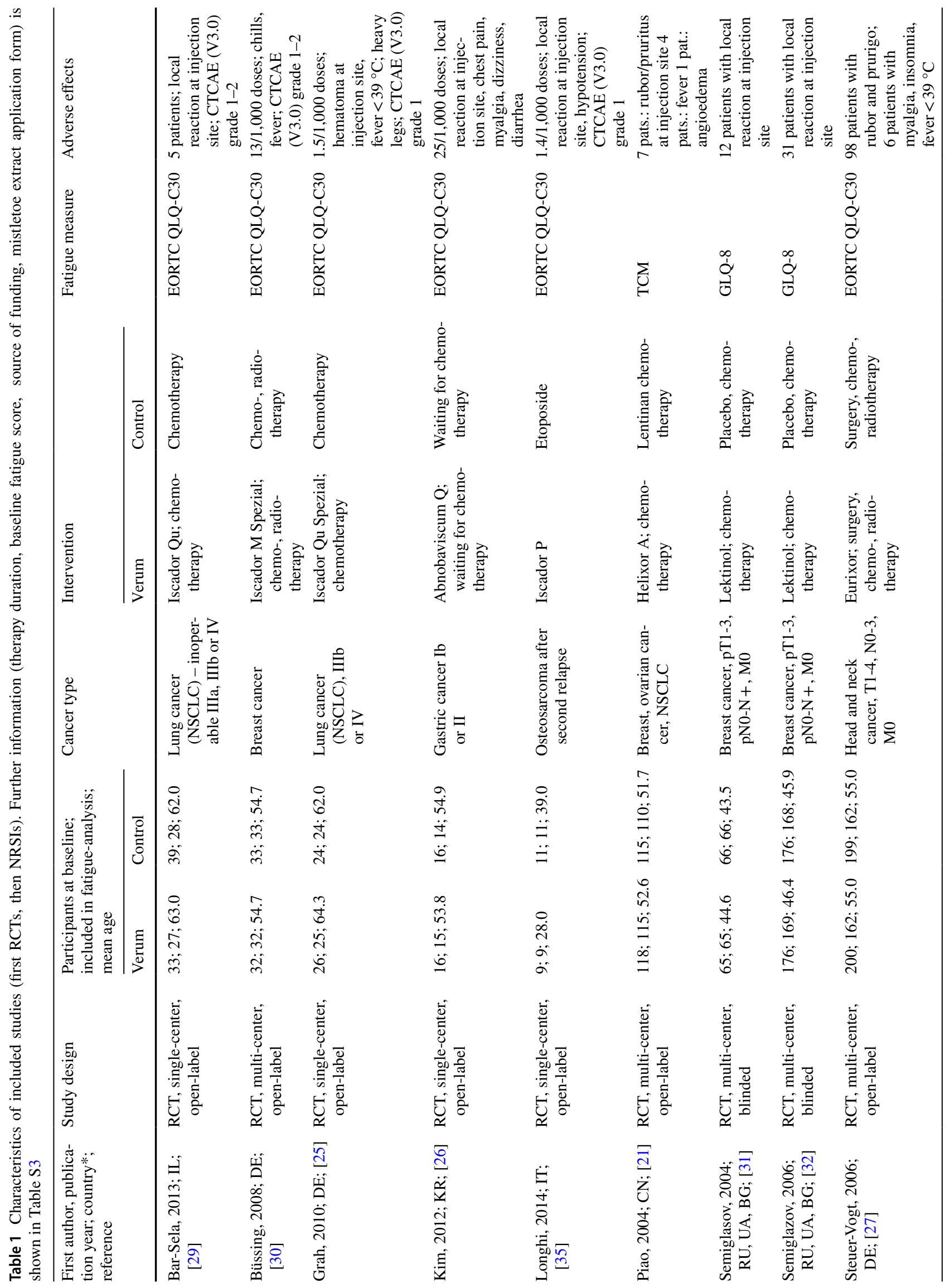




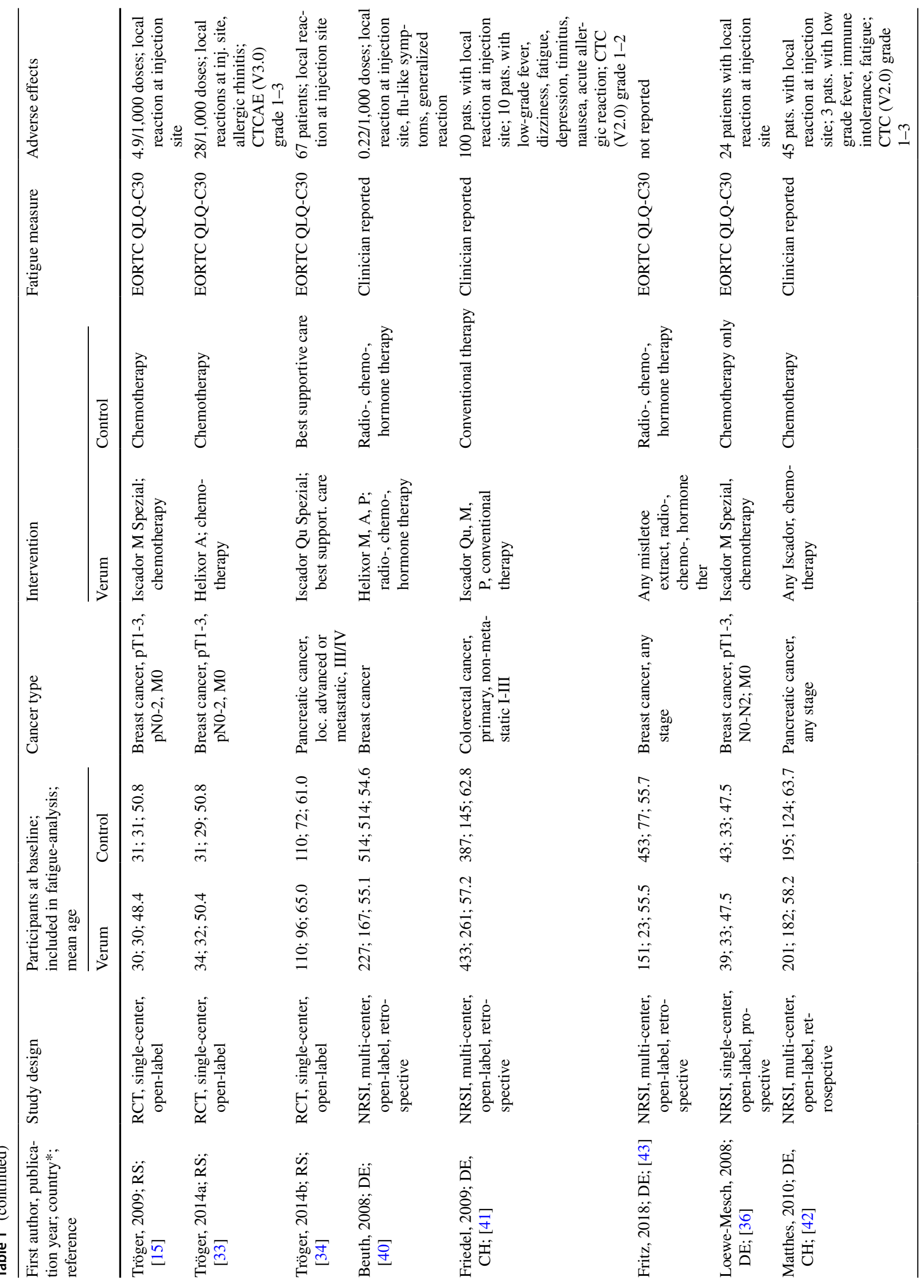




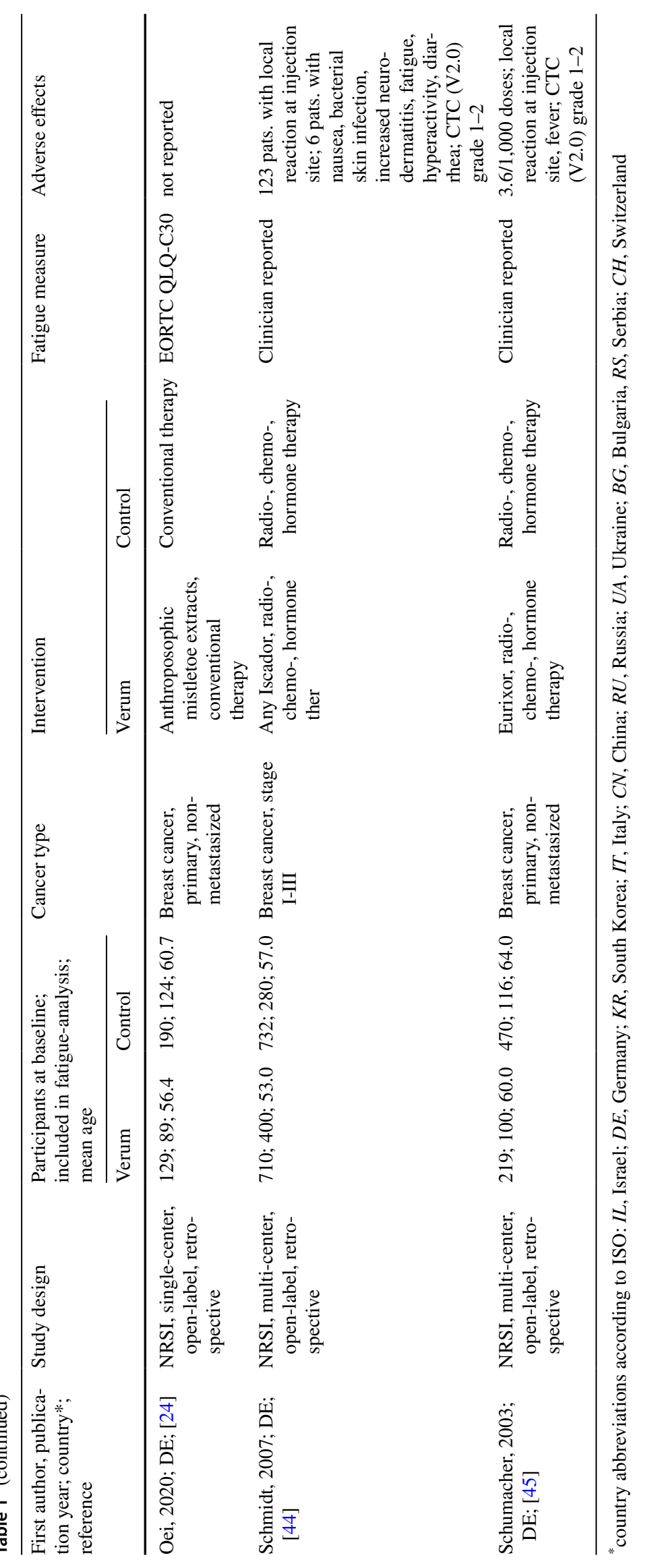


a group of mixed cancer types (breast, ovarian, and lung) were assessed in one study each. Mistletoe extracts were given in addition to conventional oncological treatments in all but two studies (Table 1).

\section{Control conditions}

The control group received placebo or active control in four trials, and only the treatment common to both arms in 16 trials (Table 1).

\section{Outcome classification and measurement}

CRF was measured with quality of life questionnaires in fifteen studies (Table 1). The quality of life questionnaires were the primary outcome in seven studies, the secondary outcome in six studies, while outcome classification was unspecified in two studies (Table S3). The questionnaires used were the EORTC QLQ-C30, the GLQ-8, and the Traditional Chinese Medicine (TCM) index in twelve, two, and one studies, respectively. Five NRSIs measured fatigue as an event of clinician reports (Table 1).

\section{Adverse effects}

The Cochrane Handbook for Systematic Reviews of Interventions defines adverse effects (AEs) as adverse events for which the causal relationship between the intervention and the event is at least a reasonable possibility [20]. AEs were reported in 18 of 20 studies (Table 1). The mean hazard rate was $1.20 \pm 0.19$ (standard deviation) AE per 1000 prescribed doses. Nine studies that reported AE severity had a mean hazard rate of $1.06 \pm 0.25 \mathrm{AE}$ grades $1-2$ per 1000 prescribed doses and $0.099 \pm 0.034 \mathrm{AE}$ grade 3 per 1000 prescribed doses. All grade $3 \mathrm{AE}$ were local reactions at the injection site. Most often reported adverse effects were local reactions at the injection site, which occurred in a mean of $24.90 \% \pm 2.90 \%$ of participants. Systemic adverse effects included fever, flu-like symptoms, nausea, diarrhea, hypotension, and acute allergic reaction and occurred in a mean of $1.83 \% \pm 0.16 \%$ of participants.

\section{Randomized controlled trials}

The results of the meta-analysis pooling the effect estimates from RCTs comparing mistletoe extracts and control with regard to CRF are presented in Fig. 2. Due to high heterogeneity $\left(I^{2}=89 \%\right)$, a random-effect meta-analysis was used to estimate a SMD of -0.48 (95\% CI -0.82 to $-0.14 ; p=0.006)$. The subgroup analyses are displayed in Table S4: 20 of 23 pooled point estimates lie within the confidence interval of the overall SMD in Fig. 2. Fourteen of 23 SMDs were significant with $p \leq 0.05$.

Meta-regressions on the impact of baseline fatigue and intervention duration showed tendencies corresponding to the results of the subgroup analysis but otherwise did not lead to reliable results due to the insufficient number of trials (data shown in supplement).

Figure 3 displays the results of the risk of bias assessment of the RCTs with the RoB 2 instrument. Of all trials, $92 \%$ had a high overall risk of bias (Fig. S3), out of which 83\% are in the domain "measurement of the outcome" (Fig. S3): high risk of bias in this domain was due to the open-label measurement of CRF in eight studies [15, 25-27, 29, 30, 33, 34] and the assessment of general aches and pain within the fatigue/tiredness dimension in two studies [31,32].

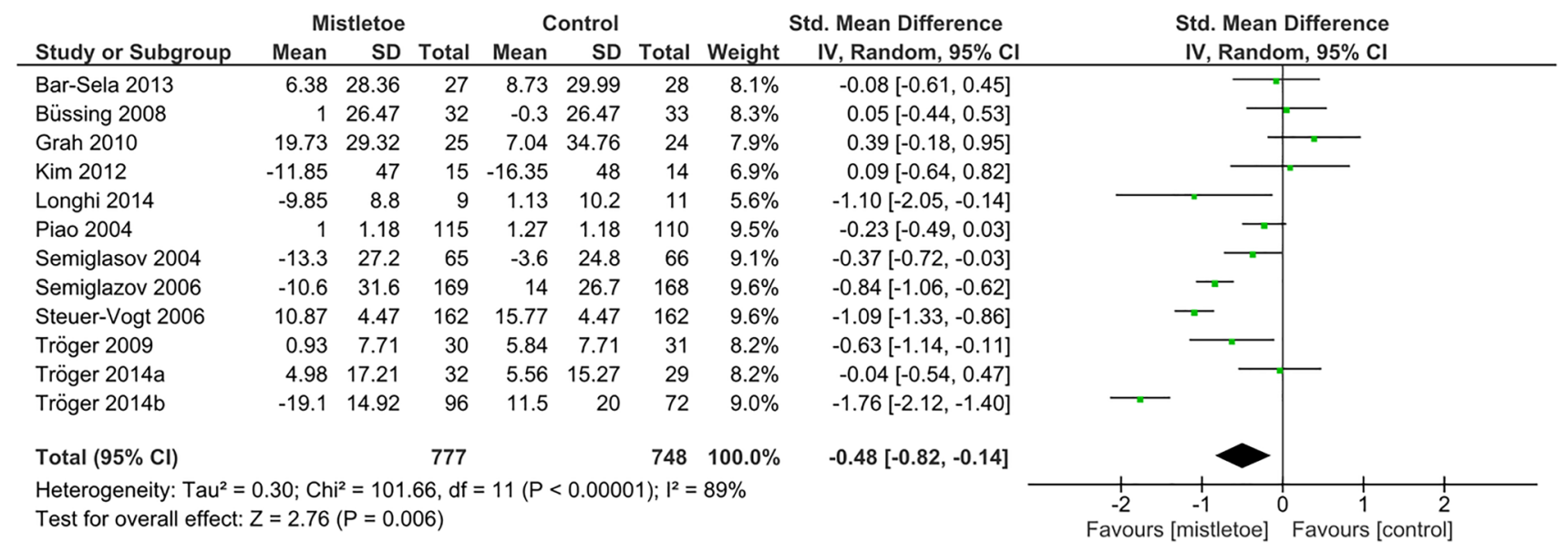

Fig. 2 Random-effect meta-analysis pooling standardized mean differences from RCTs regarding the effect of mistletoe extracts vs. control on cancer-related fatigue 
Fig. 3 Details of risk of bias assessment of RCTs according to Cochrane RoB 2 tool (intention-to-treat). Treatments include chemotherapy (CTX), therapy with mistletoe extracts (ME), and best supportive care (BSC)

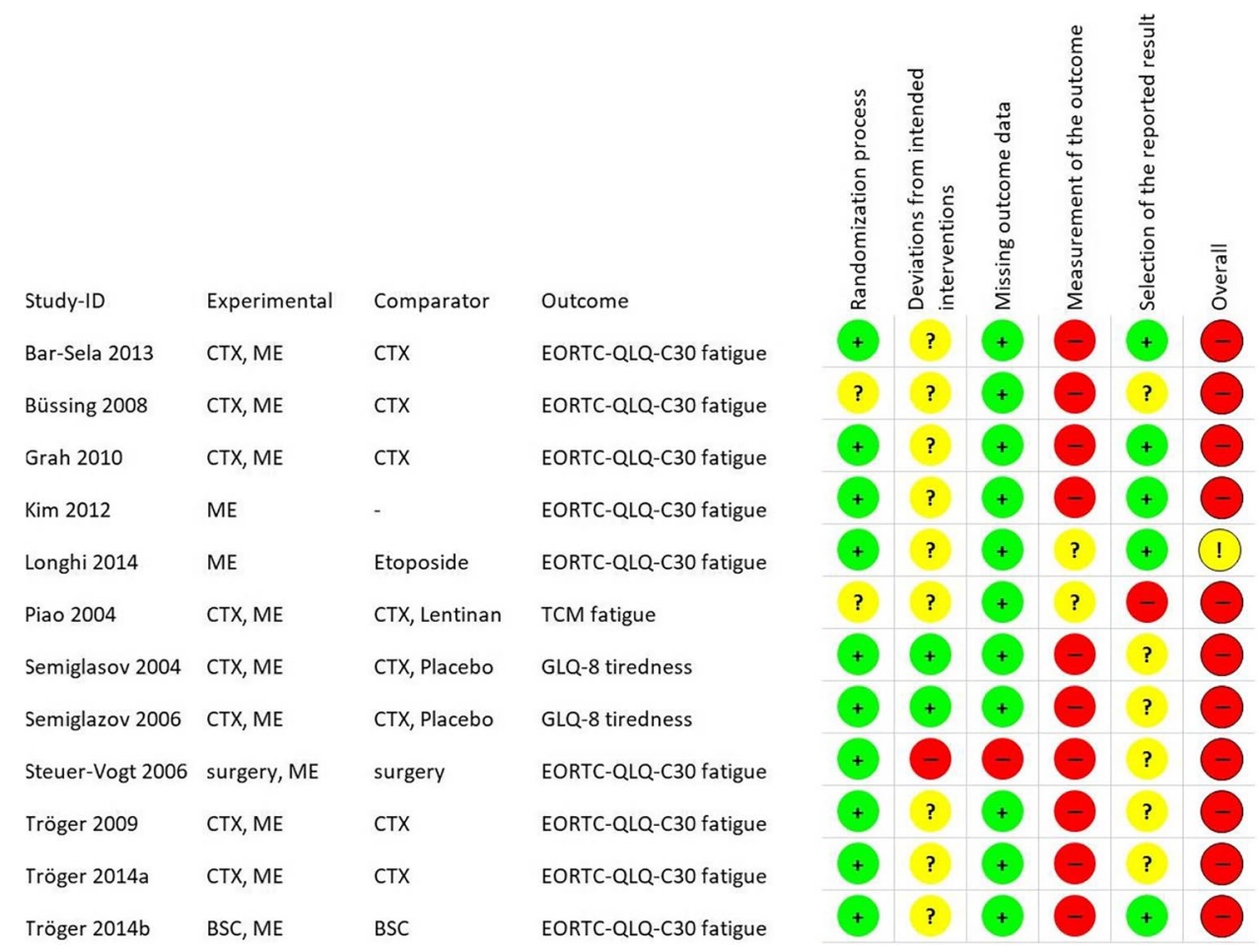

\section{Non-randomized studies of interventions}

To avoid pooling prospective and retrospective designs, the prospective NRSI was included only in sensitivity analyses, shown in the supplementary data. The meta-analysis of retrospective NRSIs is displayed in Fig. 4. Due to high heterogeneity $\left(I^{2}=77 \%\right)$, a random-effect meta-analysis was used to estimate an OR of 0.36 (95\% CI 0.20 to $0.66 ; p=0.0008$ ). The corresponding subgroup analyses are presented in Table S5. With the exception of NRSIs that used EORTC QLQC30 [24, 43], the pooled point estimates of all subgroup analyses are within the confidence interval of the overall OR in Fig. 4.

Figure 5 shows the risk of bias assessment of NRSIs with the ROBINS-I tool. The overall risk of bias was serious for all studies. All studies were judged to be at serious risk of bias in at least two domains, but not at critical risk of bias in any domain.

\section{Sensitivity analysis}

The overall meta-analysis regarding RCTs (Fig. 2) was repeated with alternative approaches regarding the handling of data multiplicity, study inclusion, and the analysis method (Table S6). The effect estimates proved robust against all alterations: neither the use of single post outcome values instead of mean effect estimates nor the application of a fixed-effect model resulted in a substantial impact on the effect magnitude or its significance. Most noteworthy, if the subgroup analyses were repeated with a fixed-effect model, the pooled effect sizes shifted towards the larger studies, which have a larger effect size (Table S7).

For NRSIs, multiple sensitivity analyses (Table S8) showed no relevant impact of alternative assumptions on the pooled effect estimate of the overall meta-analysis (Fig. 4).

The additional inclusion of a prospective NRSI [36] did not substantially alter the respective pooled effect sizes
Fig. 4 Random-effect metaanalysis pooling odds ratios from retrospective NRSIs regarding the effect of mistletoe extracts vs. control on cancer-related fatigue. OR were adjusted for baseline fatigue $[24,40,43]$ or multiply adjusted

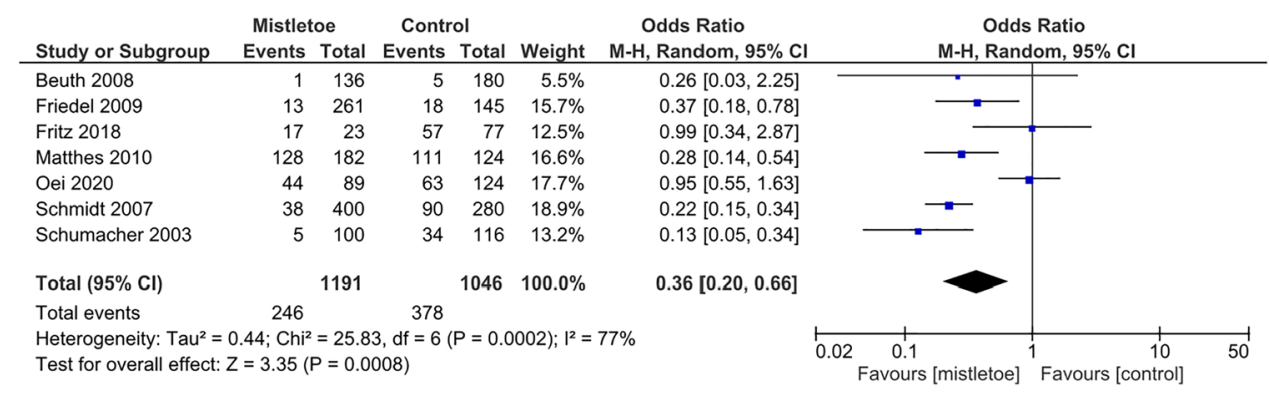


Fig. 5 Risk of bias assessment of NRSIs according to Cochrane ROBINS-I tool (intention-totreat)

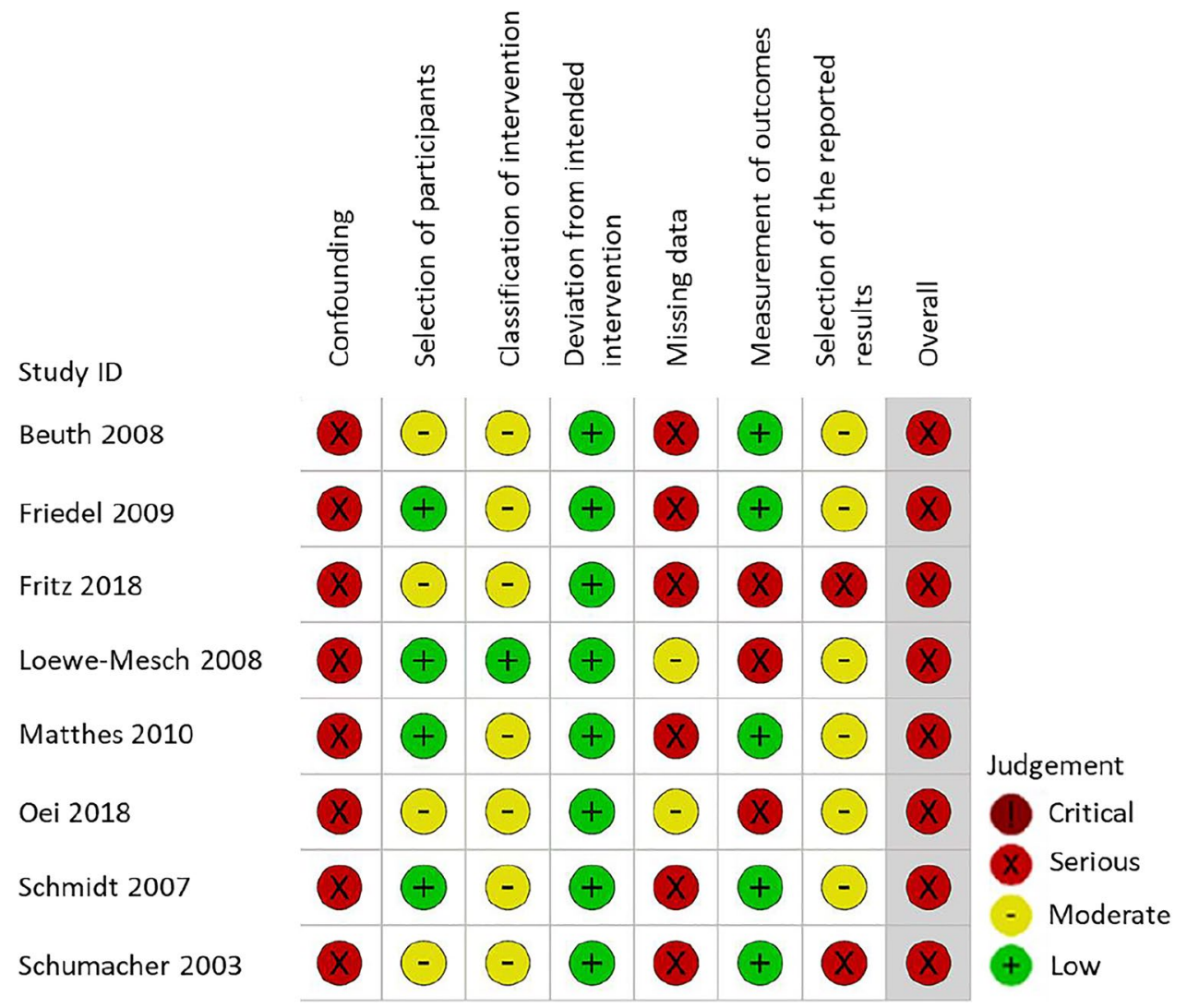

neither in the evaluation of all prospective studies (together with RCTs) nor in the analysis of all observational studies (together with the retrospective NRSIs) (Table S6 and S8).

\section{Publication bias}

The meta-analysis pooling RCTs was examined for publication bias in three ways. First, the funnel plot does not resemble a symmetrical inverted funnel (Fig. S4). Second, Egger's test displays an intercept of 3.01 (95\% CI -1.16 to 7.18; $p=0.187)$. Third, Duval and Tweedie's trim-and-fill procedure estimates no studies to be omitted on the right and three studies to be added on the left side (Fig. S5), leading to an effect estimate of SMD $=-0.70(95 \% \mathrm{CI}-1.12$ to $-0.29, p=0.0025$ ).

For NRSIs, the studies are not symmetrically distributed in the funnel plot (Fig. S6).

\section{Discussion}

Mistletoe extracts reduce CRF compared to control in RCTs and NRSIs. In RCT, the pooled effect estimate was SMD $=-0.48$, which represents a moderate effect [46]. In NRSIs, the pooled effect estimate was $\mathrm{OR}=0.36$, which in a numeric sense represents a moderate to large effect [47].
To our knowledge, the effect of mistletoe extracts on CRF has previously only been investigated as a subgroup analysis within a meta-analysis on quality of life [17]. Our results show a statistically significant but smaller effect size than the previous meta-analysis, which included 9 RCTs with 779 patients and calculated an SMD of -0.79 (95\% CI -1.66 to $-0.08, p=0.08$ ).

The results are robust, despite a high between-study heterogeneity. The high heterogeneity possibly derives from differences regarding the study population (e.g., cancer types, baseline fatigue levels) and methodological variations (e.g., mistletoe extract type, blinding, intervention duration, CRF measurement). Investigation of heterogeneity is difficult when there are few studies [20]. We opted to follow a pragmatic way [20] by calculating random-effect meta-analyses and testing the impact of the alternative use of fixed-effect models in the sensitivity analysis. This had no substantial influence on the SMD or the OR calculated in the overall analyses. This robustness is additionally supported by the subgroup analyses which resulted in SMDs and ORs of magnitudes that were similar to the overall pooled effect estimates.

The results have several limitations, however. Firstly, the subgroups' explanatory power should be interpreted with caution. The number of included studies and participants often differed for subgroups of a given moderator; the 
uneven covariate distribution may limit the usefulness of the findings [48, 49]. In addition, there was neither clear consensus between the results of the subgroup analyses in the RCT group and two meta-regressions nor between the subgroup analyses in the RCT and the NRSI groups. This lack of consensus might be partly caused by the pooling of studies with small sample sizes [50]. For this reason, and since thresholds of the $I^{2}$ statistic can be misleading for choosing a meta-analytical model [20], we tested the reliability of the subgroup SMDs by applying the fixed instead of the random-effect model. The effect estimates shifted towards the larger studies during the fixed-effect meta-analysis, indicating a smallstudy effect [51]. This and our subgroup analysis on sample size indicate that the effect estimates are different between small and larger trials. The small study effect should not be seen as a publication bias, however, as explained below.

Secondly, the risk of bias was high for 11 of 12 RCTs and serious for all NRSIs. In RCTs, the most prevalent high risk of bias is related to the lack of blinding. The lack of blinding is unlikely to affect the CRF measurement in RCTs, however. A recent meta-epidemiological study suggests that missing blinding has no substantial impact on treatment effect [52] and thereby cautiously questions two meta-analyses suggesting a placebo effect in CRF-treatment [53, 54]. In NRSIs, on the other hand, a recurrent serious risk of bias is observed in the domains for confounding. Numerous potential confounders for CRF have not been recorded, some because they were not known at the time of the study, e.g., physical exercise before the year 2005 .

Thirdly, NRSIs can overestimate treatment effects e.g. due to selective dropout of patients experiencing only insufficient effectiveness [55]. To reduce this overestimation, we can adjust our OR by the factor for average overestimation according to Hemkens et al. [55]. This adjusted estimation would result in a moderate effect size [47] and indicate a positive, clinically relevant impact of individualized mistletoe extract treatment schemes on CRF.

Fourthly, publication bias can neither be confirmed nor excluded due to the low number of identified RCTs and to their heterogeneity. Egger's test and Duval and Tweedie's trim-and-fill procedure are usually recommended for a minimum of 10 studies [56]; we included 12 trials with high heterogeneity, which is why the results should be interpreted with great care [57]. While Egger's test indicates no publication bias, the visual funnel plot examination and Duval and Tweedie's trim-and-fill procedure may imply a bias, yet with contradictory tendencies.

Mistletoe extracts can be recommended within cancer care to treat CRF as an alternative or add-on therapy to physical activity. Mistletoe extracts have a pooled effect estimate that is comparable to other CRF interventions such as physical activity (SMD $=-0.30(95 \% \mathrm{CI}-0.25$ to -0.36$))$ [58], Tai Chi and Qigong (SMD $=-0.53(95 \% \mathrm{CI}-0.97$ to -0.28$)$ )
[59], and yoga (SMD $=-0.30(95 \% \mathrm{CI}-0.51$ to -0.08$)$ ) [60]. Patients would therefore have no disadvantage by choosing mistletoe extracts in addition to or instead of the aforementioned therapies, especially those who can no longer perform physical activities. The inclusion of mistletoe extracts in future multimodal treatment studies should be considered. Mistletoe extracts also have advantages compared to other pharmacological treatments. Firstly, mistletoe extracts have a higher effect size than methylphenidate $(\mathrm{SMD}=-0.28$ (95\% CI -0.44 to -0.12$)$ ) [7]. Secondly, this systematic review has found few adverse effects reported within the study durations. All reported adverse effects, such as local reactions at the injection site, are well manageable by an experienced practitioner, who should accompany treatment with mistletoe extracts. Previously published observational studies [61-63], reviews [64], and RCTs [65] identified the same types and similar hazard rates of adverse effects. Mistletoe extracts also do not seem to have a negative impact on disease-free and overall survival, but a positive one, as a recent meta-analysis showed [18].

\section{Conclusion}

Both sources of evidence, RCTs and NRSIs, lead to effect estimates that imply a significant symptom-reducing effect of mistletoe extracts against control regarding CRF. This result is relevant for healthcare providers seeking a pharmacological treatment for CRF, in oncological settings where physical activity is not possible or where complementary CRF treatment is sought.

Future RCTs assessing the effectiveness of mistletoe extracts in CRF management need to be placebo-controlled and identify CRF as the primary outcome. Suggested topics of research are the impact of the duration of mistletoe extract treatment on CRF and the impact of mistletoe extracts on CRF in post-treatment cancer survivors. Future NRSIs need to record confounders to achieve higher certainty of evidence. NRSIs remain important sources of evidence to assess the effectiveness of individualized treatments with mistletoe extracts.

Supplementary Information The online version contains supplementary material available at https://doi.org/10.1007/s00520-022-06921-x.

Author contribution All authors have contributed to the conceptualization (PROSPERO-protocol) of the meta-analysis. FP and SB have performed the literature search and selection. FP and ML have performed the data extraction, the risk of bias assessment, the statistical analysis and have set up the figures and tables. FP has written the original draft and all authors have contributed to its review and editing.

Funding Open Access funding enabled and organized by Projekt DEAL. This study was funded by the Society for Cancer Research, 
Arlesheim, Switzerland, and the Gesellschaft für klinische Forschung e.V., Berlin, Germany.

Data availability The authors state that they have full control of all primary data and agree to allow the journal to review this data if requested.

\section{Code availability N/A.}

\section{Declarations}

Ethics approval N/A.

\section{Consent to participate N/A}

\section{Consent for publication N/A}

Conflict of interest The nonprofit organizations Society for Cancer Research and Gesellschaft für klinische Forschung e.V. are minority shareholders of Iscador AG, a manufacturer of mistletoe extracts. FP is an employee of the Society for Cancer Research. SB is a part-time employee of the Society for Cancer Research and Iscador AG. ML is a researcher at the CHS Institute which received funding from the Gesellschaft für klinische Forschung e.V.. DDM has no conflict of interest.

Open Access This article is licensed under a Creative Commons Attribution 4.0 International License, which permits use, sharing, adaptation, distribution and reproduction in any medium or format, as long as you give appropriate credit to the original author(s) and the source, provide a link to the Creative Commons licence, and indicate if changes were made. The images or other third party material in this article are included in the article's Creative Commons licence, unless indicated otherwise in a credit line to the material. If material is not included in the article's Creative Commons licence and your intended use is not permitted by statutory regulation or exceeds the permitted use, you will need to obtain permission directly from the copyright holder. To view a copy of this licence, visit http://creativecommons.org/licenses/by/4.0/.

\section{References}

1. Denlinger C et al (2014) Survivorship: fatigue, version 1.2014. J Natl Compr Canc Netw 12:876-887. https://doi.org/10.6004/ jnccn.2014.0082

2. Al Maqbali M et al (2019) Quality assessment criteria: psychometric properties of measurement tools for cancer related fatigue. Acta Oncol 58:1286-1297. https://doi.org/10.1080/0284186X. 2019.1622773

3. Filler K, Saligan L (2016) Defining cancer-related fatigue for biomarker discovery. Support Care Cancer 24:5-7. https://doi.org/10. 1007/s00520-015-2965-5

4. Barsevick A et al (2010) ASCPRO recommendations for the assessment of fatigue as an outcome in clinical trials. J Pain Symptom Manage 39:1086-1099. https://doi.org/10.1016/j.jpain symman.2010.02.006

5. Al Maqbali M et al (2021) Prevalence of fatigue in patients with cancer: a systematic review and meta-analysis. J Pain Symptom Manage 61:167-189. https://doi.org/10.1016/j.jpainsymman. 2020.07.037

6. Fabi A et al (2020) Cancer-related fatigue: ESMO Clinical Practice Guidelines for diagnosis and treatment. Ann Oncol 31:713723. https://doi.org/10.1016/j.annonc.2020.02.016
7. Qu D et al (2016) Psychotropic drugs for the management of cancer-related fatigue: a systematic review and meta-analysis. Eur J Cancer Care 25:970-979. https://doi.org/10.1111/ecc.12397

8. Kelley G, Kelley K (2017) Exercise and cancer-related fatigue in adults: a systematic review of previous systematic reviews with meta-analyses. BMC Cancer 17:693. https://doi.org/10.1186/ s12885-017-3687-5

9. Thong M et al (2020) Cancer-related fatigue: causes and current treatment options. Curr Treat Options Oncol 21:17. https://doi. org/10.1007/s11864-020-0707-5

10. Yennurajalingam $\mathrm{S}$ et al (2021) Sleep disturbance in patients with cancer: a feasibility study of multimodal therapy. BMJ Support Palliat Care 11:170-179. https://doi.org/10.1136/bmjsp care-2019-001877

11. Kienle $\mathrm{G}$ et al (2018) The subjective dimension of integrative cancer care: a qualitative study exploring the perspectives, themes, and observations of experienced doctors from the area of anthroposophic medicine. Explore (NY) 14:342-351. https://doi.org/10. 1016/j.explore.2018.03.007

12. National Cancer Institute USA (2020) Mistletoe Extracts (PDQ®)-Health Professional Version. PDQ Cancer Information Summaries https://www.cancer.gov/about-cancer/treatment/cam/ hp/mistletoe-pdq Accessed 28 July 2020.

13. Horneber $\mathrm{M}$ et al (2008) Mistletoe therapy in oncology. Cochrane Database Syst Rev 2:CD003297. https://doi.org/10.1002/14651 858.CD003297.pub2

14. Oei S, Thronicke A, Schad F (2019) Mistletoe and immunomodulation: insights and implications for anticancer therapies. Evid Based Complement Alternat Med 2019:5893017. https://doi.org/ $10.1155 / 2019 / 5893017$

15. Tröger W et al (2009) Quality of life and neutropenia in patients with early stage breast cancer: a randomized pilot study comparing additional treatment with mistletoe extract to chemotherapy alone. Breast Cancer (Auckl) 3:35-45. https://doi.org/10.4137/ bcbcr.s2905

16. Kienle $\mathrm{G}$ et al (2016) Individualized Integrative Cancer Care in Anthroposophic Medicine: A Qualitative Study of the Concepts and Procedures of Expert Doctors. Integr Cancer Ther 15:478 494. https://doi.org/10.1177/1534735416640091

17. Loef M, Walach H (2020) Quality of life in cancer patients treated with mistletoe: a systematic review and meta-analysis. BMC Complement Med Ther 20:227. https://doi.org/10.1186/ s12906-020-03013-3

18. Ostermann $\mathrm{T}$ et al (2020) A systematic review and meta-analysis on the survival of cancer patients treated with a fermented Viscum album L. Extract (Iscador): an update of findings. Complement Med Res 27:260-271. https://doi.org/10.1159/000505202

19. Liberati A et al (2009) The PRISMA statement for reporting systematic reviews and meta-analyses of studies that evaluate health care interventions: explanation and elaboration. PLoS Med 6:e1000100. https://doi.org/10.1371/journal.pmed.1000100

20. Higgins JPT et al (2020) Cochrane Handbook for Systematic Reviews of Interventions version 6.1. https://training.cochrane. org/handbook. Accessed 28 April 2020

21. Piao B et al (2004) Impact of complementary mistletoe extract treatment on quality of life in breast, ovarian and non-small cell lung cancer patients. A prospective randomized controlled clinical trial. Anticancer Res 24:303-309

22. Hasselblad V, Hedges L (1995) Meta-analysis of screening and diagnostic tests. Psychol Bull 117:167. https://doi.org/10.1037/ 0033-2909.117.1.167

23. Chinn S (2000) A simple method for converting an odds ratio to effect size for use in meta-analysis. Stat Med 19:3127-3131. https://doi.org/10.1002/1097-0258(20001130)19:22\%3c3127:: aid-sim784\%3e3.0.co;2-m 
24. Oei S et al (2020) Impact of oncological therapy and Viscum album L treatment on cancer-related fatigue and internal coherence in nonmetastasized breast cancer patients. Integr Cancer Ther 19:1-16. https://doi.org/10.1177/1534735420917211

25. Grah C (2010) Misteltherapie bei nichtkleinzelligem Bronchialkarzinom. Dissertation, Charité - Universitätsmedizin Berlin

26. Kim K et al (2012) Quality of life, immunomodulation and safety of adjuvant mistletoe treatment in patients with gastric carcinoma - a randomized, controlled pilot study. BMC Complement Altern Med 12:172. https://doi.org/10.1186/1472-6882-12-172

27. Steuer-Vogt M et al (2006) Influence of ML-1 standardized mistletoe extract on the quality of life in head and neck cancer patients. HNO 54:277-286. https://doi.org/10.1007/s00106-005-1318-y

28. López-López J et al (2018) Dealing with effect size multiplicity in systematic reviews and meta-analyses. Res Synth methods 9:336-351. https://doi.org/10.1002/jrsm.1310

29. Bar-Sela G et al (2013) Mistletoe as complementary treatment in patients with advanced non-small-cell lung cancer treated with carboplatin-based combinations: a randomised phase II study. Eur J Cancer 49:1058-1064. https://doi.org/10.1016/j.ejca.2012.11. 007

30. Büssing A et al (2008) Modulation of chemotherapy-associated immunosuppression by intravenous application of Viscum album L. extract (Iscador): a randomised phase II study. Eur J Integr Med 1:2-3. https://doi.org/10.1016/j.eujim.2008.08.084

31. Semiglasov V et al (2004) The standardised mistletoe extract PS76A2 improves QoL in patients with breast cancer receiving adjuvant $\mathrm{CMF}$ chemotherapy: a randomised, placebo-controlled, double-blind, multicentre clinical trial. Anticancer Res 24:1293-1302

32. Semiglazov V et al (2006) Quality of life is improved in breast cancer patients by Standardised Mistletoe Extract PS76A2 during chemotherapy and follow-up: a randomised, placebo-controlled, double-blind, multicentre clinical trial. Anticancer Res 26:1519-1529

33. Tröger $\mathrm{W}$ et al (2014) Additional therapy with a mistletoe product during adjuvant chemotherapy of breast cancer patients improves quality of life: an open randomized clinical pilot trial. Evid Based Complement Alternat Med 2014:430518. https://doi.org/10.1155/ 2014/430518

34. Tröger W et al (2014) Quality of life of patients with advanced pancreatic cancer during treatment with mistletoe: a randomized controlled trial. Dtsch Arztebl Int 111:493-502. https://doi.org/ 10.3238/arztebl.2014.0493

35. Longhi A et al (2014) A randomized study on postrelapse disease-free survival with adjuvant mistletoe versus oral etoposide in osteosarcoma patients. Evid Based Complement Alternat Med 2014:210198. https://doi.org/10.1155/2014/210198

36. Loewe-Mesch A et al (2008) Adjuvante simultane Mistel-/Chemotherapie bei Mammakarzinom-Einfluss auf Immunparameter, Lebensqualität und Verträglichkeit. Complement Med Res 15:22-30. https://doi.org/10.1159/000112860

37. Higgins J, Thompson S (2002) Quantifying heterogeneity in a meta-analysis. Stat Med 21:1539-1558. https://doi.org/10.1002/ sim. 1186

38. Egger $\mathrm{M}$ et al (1997) Bias in meta-analysis detected by a simple, graphical test. Br Med J 315:629-634. https://doi.org/10.1136/ bmj.315.7109.629

39. Duval S, Tweedie R (2000) Trim and fill: a simple funnel-plotbased method of testing and adjusting for publication bias in metaanalysis. Biometrics 56:455-463. https://doi.org/10.1111/j.0006341x.2000.00455.x

40. Beuth J, Schneider B, Schierholz J (2008) Impact of complementary treatment of breast cancer patients with standardized mistletoe extract during aftercare: a controlled multicenter comparative epidemiological cohort study. Anticancer Res 28:523-527
41. Friedel W et al (2009) Systematic evaluation of the clinical effects of supportive mistletoe treatment within chemo-and/or radiotherapy protocols and long-term mistletoe application in nonmetastatic colorectal carcinoma: multicenter, controlled, observational cohort study. J Soc Integr Oncol 7:137-145. https://doi.org/10.2310/7200.2009.0014

42. Matthes $\mathrm{H}$ et al (2010) Molecular mistletoe therapy: friend or foe in established anti-tumor protocols? A multicenter, controlled, retrospective pharmaco-epidemiological study in pancreas cancer. Curr Mol Med 10:430-439. https://doi.org/10. 2174/156652410791317057

43. Fritz $\mathrm{P}$ et al (2018) Is mistletoe treatment beneficial in invasive breast cancer? A new approach to an unresolved problem. Anticancer Res 38:1585-1593. https://doi.org/10.21873/anticanres. 12388

44. Schmidt N (2007) Die postoperative komplementäre Therapie des primären Mammakarzinom mit lektinnormiertem Mistelextrakt - eine epidemiologische, kontrollierte retrolektive Kohortenstudie. Dissertation, Charité - Universitätsmedizin Berlin

45. Schumacher K et al (2003) Influence of postoperative complementary treatment with lectin-standardized mistletoe extract on breast cancer patients. A controlled epidemiological multicentric retrolective cohort study. Anticancer Res 23:5081-5087

46. Cohen J (1988) Statistical power analysis for the behavioral sciences, 2nd edn. Lawrence Erlbaum Associates, Hillsdale, NJ

47. Rahlfs V, Zimmermann H (2019) Effect size measures and their benchmark values for quantifying benefit or risk of medicinal products. Biom J 61:973-982. https://doi.org/10.1002/bimj. 201800107

48. Richardson M, Garner P, Donegan S (2019) Interpretation of subgroup analyses in systematic reviews: a tutorial. Clin Epidemiol Glob Health 7:192-198. https://doi.org/10.1016/j.cegh. 2018.05.005

49. Donegan $\mathrm{S}$ et al (2015) Exploring treatment by covariate interactions using subgroup analysis and meta-regression in cochrane reviews: a review of recent practice. PloS One 10:e0128804. https://doi.org/10.1371/journal.pone.0128804

50. Bakbergenuly I, Hoaglin D, Kulinskaya E (2020) Estimation in meta-analyses of mean difference and standardized mean difference. Stat Med 39:171-191. https://doi.org/10.1002/sim.8422

51. Sterne J, Gavaghan D, Egger M (2000) Publication and related bias in meta-analysis: power of statistical tests and prevalence in the literature. J Clin Epidemiol 53:1119-1129. https://doi. org/10.1016/s0895-4356(00)00242-0

52. Moustgaard $\mathrm{H}$ et al (2020) Impact of blinding on estimated treatment effects in randomised clinical trials: meta-epidemiological study. Br Med J 368:16802. https://doi.org/10.1136/ bmj.16802

53. Aguiar P et al (2020) The efficacy of placebo for the treatment of cancer-related fatigue: a systematic review and meta-analysis. Support Care Cancer 28:1755-1764. https://doi.org/10.1007/ s00520-019-04977-w

54. Roji R et al (2020) Placebo response in trials of drug treatments for cancer-related fatigue: a systematic review, meta-analysis and meta-regression. BMJ Support Palliat Care 10:385-394. https://doi.org/10.1136/bmjspcare-2019-002163

55. Hemkens L, Contopoulos-Ioannidis D, Ioannidis J (2016) Agreement of treatment effects for mortality from routinely collected data and subsequent randomized trials: meta-epidemiological survey. Br Med J 352:i493. https://doi.org/10.1136/ bmj.i493

56. Sterne J et al (2011) Recommendations for examining and interpreting funnel plot asymmetry in meta-analyses of randomised controlled trials. Br Med J 343:d4002. https://doi.org/10.1136/ bmj.d4002 
57. Peters $\mathrm{J}$ et al (2007) Performance of the trim and fill method in the presence of publication bias and between-study heterogeneity. Stat Med 26:4544-4562. https://doi.org/10.1002/sim.2889

58. Mustian K et al (2017) Comparison of pharmaceutical, psychological, and exercise treatments for cancer-related fatigue: a metaanalysis. JAMA Oncol 3:961-968. https://doi.org/10.1001/jamao ncol.2016.6914

59. Wayne P et al (2018) Tai Chi and Qigong for cancer-related symptoms and quality of life: a systematic review and metaanalysis. J Cancer Surviv 12:256-267. https://doi.org/10.1007/ s11764-017-0665-5

60. O'Neill M et al (2020) The effect of yoga interventions on cancerrelated fatigue and quality of life for women with breast cancer: a systematic review and meta-analysis of randomized controlled trials. Integr Cancer Ther 19:1-10. https://doi.org/10.1177/15347 35420959882

61. Schad F et al (2018) Safety of combined treatment with monoclonal antibodies and Viscum album $L$ preparations. Integr Cancer Ther 17:41-51. https://doi.org/10.1177/1534735416681641

62. Steele $\mathrm{M}$ et al (2014) Adverse drug reactions and expected effects to therapy with subcutaneous mistletoe extracts (Viscum album
L.) in cancer patients. Evid Based Complement Alternat Med 2014:724258. https://doi.org/10.1155/2014/724258

63. Thronicke A et al (2018) Clinical safety of combined targeted and Viscum album L. therapy in oncological patients. Medicines 5:100. https://doi.org/10.3390/medicines5030100

64. Melzer J et al (2009) Efficacy and safety of mistletoe preparations (Viscum album) for patients with cancer diseases: A systematic review. Complement Med Res 16:217-226. https://doi.org/10. $1159 / 000226249$

65. Pelzer F, Tröger W (2018) Complementary treatment with mistletoe extracts during chemotherapy: safety, neutropenia, fever, and quality of life assessed in a randomized study. J Altern Complement Med 24:954-961. https://doi.org/10.1089/acm.2018.0159

Publisher's Note Springer Nature remains neutral with regard to jurisdictional claims in published maps and institutional affiliations. 